This item was submitted to Loughborough's Research Repository by the author.

Items in Figshare are protected by copyright, with all rights reserved, unless otherwise indicated.

\title{
Hydrodynamic interactions dominate the structure of active swimmers' pair distribution functions
}

PLEASE CITE THE PUBLISHED VERSION

https://doi.org/10.1063/1.5085755

\section{PUBLISHER}

(c) the Authors. Published by AIP Publishing

\section{VERSION}

AM (Accepted Manuscript)

\section{PUBLISHER STATEMENT}

This article may be downloaded for personal use only. Any other use requires prior permission of the author and AIP Publishing. This article appeared in SCHWARZENDAHL, F.J. and MAZZA, M.G., 2019.

Hydrodynamic interactions dominate the structure of active swimmers' pair distribution functions. Journal of Chemical Physics, 150: 184902 and may be found at https://doi.org/10.1063/1.5085755

\section{LICENCE}

\section{All Rights Reserved}

\section{REPOSITORY RECORD}

Schwarzendahl, Fabian J., and Marco Mazza. 2019. "Hydrodynamic Interactions Dominate the Structure of Active Swimmers' Pair Distribution Functions”. Loughborough University. https://hdl.handle.net/2134/37852. 


\title{
Hydrodynamic interactions dominate the structure of active swimmers' pair distribution functions
}

\author{
Fabian Jan Schwarzendahl ${ }^{1}$ and Marco G. Mazza ${ }^{2,1}$ \\ 1) Max Planck Institute for Dynamics and Self-Organization, Am Faßberg 17, 37077 Göttingen, \\ Germany \\ ${ }^{2)}$ Interdisciplinary Centre for Mathematical Modelling and Department of Mathematical Sciences, \\ Loughborough University, Loughborough, Leicestershire LE11 3TU, United Kingdom
}

(Dated: 2 April 2019)

\begin{abstract}
Microswimmers often exhibit surprising patterns due to the nonequilibrium nature of their dynamics. Collectively, suspensions of microswimmers appear as a liquid whose properties set it apart from its passive counterpart. To understand the impact of hydrodynamic interactions on the basic statistical features of a microswimmer's liquid, we investigate its structure by means of the pair distribution function. We perform particle-based simulations of microswimmers that include steric effects, shape anisotropy, and hydrodynamic interactions. We find that hydrodynamic interactions considerably alter the orientation-dependent pair distribution function compared to purely excluded-volume models like active Brownian particles, and generally decrease the structure of the liquid. Depletion regions are dominant at lower filling fractions, while at larger filling fraction the microswimmer liquid develops a stronger first shell of neighbors in specific directions, while losing structure at larger distances. Our work is a first step towards a statistico-mechanical treatment of the structure of microswimmer suspensions.
\end{abstract}

\section{INTRODUCTION}

Collections of microorganisms such as bacteria or microalgae show a myriad of self-organized structures and patterns that are also governed by physical processes stemming from their nonequilibrium nature and mutual interactions. Some examples are selfconcentration $^{1,2}$, complex interaction with solid surfaces $^{3-5}$, bacterial turbulence ${ }^{6}$, swarming ${ }^{7}$, spontaneous formation of spiral vortices ${ }^{8}$, intriguing effects of hydrodynamic interactions $^{9-11}$, and directed motion ${ }^{12}$. Many of these systems collectively resemble the behavior and structure of a fluid and therefore it has been found useful to model these collections of organisms as an active or living fluid ${ }^{13,14}$. Furthermore, the methods of statistical mechanics of classical fluids have been found to be powerful tools also in nonequilibrium situations, such as the analysis of velocity correlations and energy spectra for mesoscale turbulence of bacteria ${ }^{6}$, and even a local equilibrium Maxwell-Boltzmann approximation for sedimenting active colloids ${ }^{15}$, later derived from a FokkerPlanck equation ${ }^{16}$.

The structure of an equilibrium fluid is typically analyzed in terms of the pair distribution function ${ }^{17,18}$, and is a cornerstone of liquid state theory ${ }^{19}$. For active fluids, some work on the structural properties in terms of pair distribution functions has been put forward ${ }^{20-31}$. An important finding is the anisotropic form of the pair correlation function of active Brownian particles (ABP) presented in $^{20-23}$, which arises from their self-propulsion.

Typically, pair correlation functions are strongly influenced by the mutual interactions between particles, which for microswimmers such as bacteria or algae moving in an aqueous milieu include hydrodynamic interactions. In this work, we analyze the influence of hydrodynamic interactions on the orientation-dependent pair distribution function of active swimmers by considering a recent model for biological swimmers, which also includes shape anisotropy and steric effects ${ }^{1}$. In order to disentangle the effects of activity, hydrodynamic and steric interactions, we compare the pair distribution functions for our model to the case of ABP (for which hydrodynamic effects are absent). We find that hydrodynamic interactions introduce considerably more structure to the pair distribution function at low filling fractions and suppress structures at high filling fractions.

The rest of this work is organized as follows. Section II introduces our model and the setup of the numerical simulations. In Sec. III we discuss the orientation-dependent pair distribution function, and its angular average: the radial distribution function. Section IV presents the distribution functions for both our swimmer's model and for ABP. Finally, in Sec. V we collect our conclusions.

\section{MODELS AND PARAMETERS}

\section{A. Stroke-averaged biological microswimmer}

We employ the stroke-averaged biological microswimmer model presented in ${ }^{1}$, which consists of an asymmetric dumbbell. Figure 1(a) shows a schematic representation of the puller-type model swimmer mimicking a Chlamydomonas reinhardtii cell, while Fig. 1(b) shows a schematic of the pusher-type model swimmer representing an Escherichia coli cell. In both cases, the small green sphere, marked with (1), represents the swimmer body and the large sphere, marked with (2), represents the stroke-average region spanned by the flagellar motion. The rigid motion of the dumbbells is governed by Newton's equations and implemented via quaternion dynamics (for details see ${ }^{1}$ ). The asymmetric dumbbell 


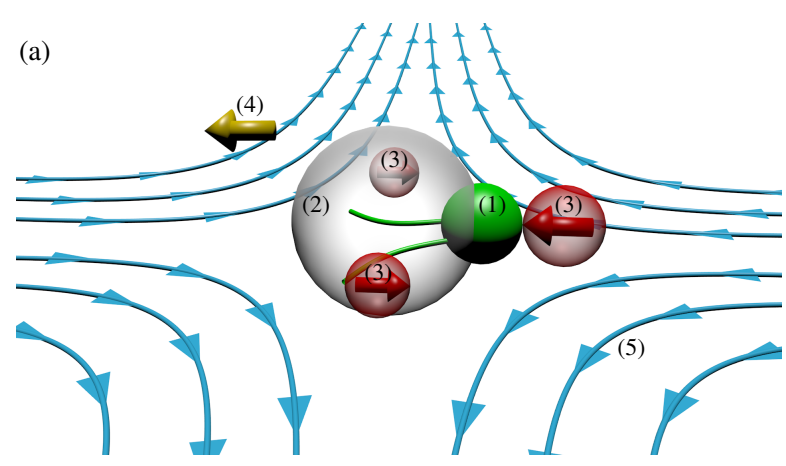

(b)

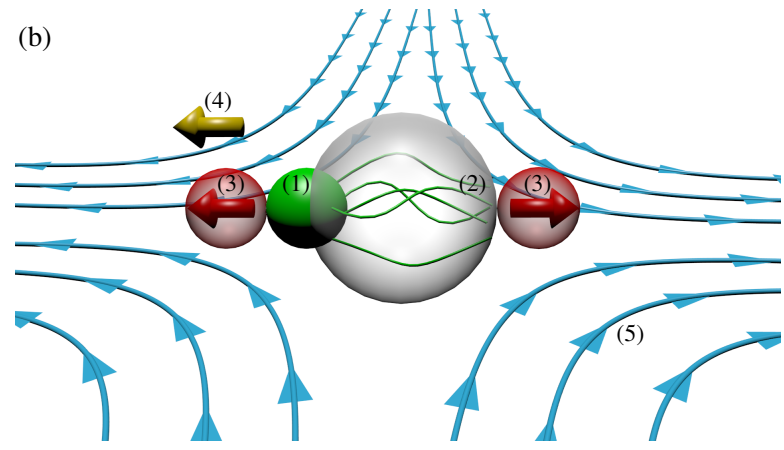

FIG. 1. Schematic of our active swimmer model (reprinted from $^{1}$ ) which shows the swimmers' composition, Stokeslets, and streamlines for a (a) puller and (b) pusher-type swimmer. For both puller and pusher, the swimmer's body is represented by the small green sphere (1), and the stroke-average of the flagella is represented by the large transparent sphere (2). The regions in which the forces are applied are shown as red spheres with embedded arrows (3). The swimming direction is shown as the golden arrow (4). The hydrodynamic streamlines are sketched as the blue lines with arrows (5).

shape of our swimmers causes the hydrodynamic center to be shifted away from the center of mass. This symmetry breaking enables the propulsion of the swimmers into the direction of the golden arrows [marked with (4) in Fig. 1].

As hydrodynamic interactions between microswimmers can play an important role in their collective behavior, we also include an explicit model for the surrounding fluid by means of the multiparticle collision dynamics (MPCD) technique. MPCD is a particle-based method that reproduces hydrodynamic modes up to the NavierStokes level of description ${ }^{32}$.

Experimental measurements ${ }^{33}$ have shown that the flow field of the pusher-type swimmer can be modeled by a force dipole, whereas puller-type swimmers' flow fields are well described by a combination of three-Stokeslets ${ }^{34}$. We use regularized force regions, as shown by the red spheres with embedded arrows (3) in Fig. 1, to account for the hydrodynamic flow fields. Additionally, the dumbbells are coupled to the fluid by using a noslip boundary condition on their surface (for more details $\left.\operatorname{see}^{1}\right)$.

The temperature $T$ of the fluid, the size of an MPCD grid cell $a$, and the mass of an MPCD particle $m$, are the

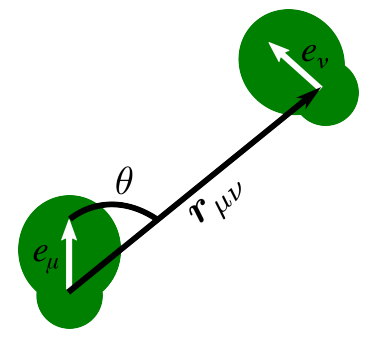

FIG. 2. Sketch of two active swimmers with relative position $\boldsymbol{r}_{\mu \nu}=\boldsymbol{r}_{\mu}-\boldsymbol{r}_{\nu}$, orientations $\boldsymbol{e}_{\mu}, \boldsymbol{e}_{\nu}$ and polar angle given by $\cos (\theta)=\boldsymbol{e}_{\mu} \cdot \boldsymbol{r}_{\mu \nu} /\left|\boldsymbol{r}_{\mu \nu}\right|$ with respect to $\boldsymbol{e}_{\mu}$. For the evaluation of the pair distribution function $g(r, \theta)$ the polar angle $\theta$ and distance $r=\left|\boldsymbol{r}_{\mu \nu}\right|$ are computed for all distinct pairs of swimmers.

reference values for temperature, length, and mass, respectively. We perform simulations in a cubic domain of size $100 a$ with periodic boundary conditions in all three directions; each MPCD cell contains an average of $\left\langle N_{\mathrm{C}}\right\rangle=20 \mathrm{MPCD}$ particles, giving a total of $2 \times 10^{7}$ MPCD particles in the system. We use the MPCD-AT+a algorithm ${ }^{32,35,36}$, which conserves the fluids' temperature with a MPCD timestep of $\delta t=0.01$. The typical length of an individual swimmer is $\sigma \approx 5 a$. For further details on the numerical implementation the reader is referred to $^{1}$.

\section{B. Active Brownian particles}

In addition to the our stroke-averaged biological microswimmer, we also carry out simulations of ABP, to disentangle the effects of activity, hydrodynamic and steric interactions. ABP are spherical particles with diameter $\sigma$ that self-propel along their orientation $\boldsymbol{e}$ with a typical speed $v_{0}$ (see also ${ }^{37-40}$ ). The translational motion of the particle's position $\boldsymbol{r}$ is governed by the equation

$$
\frac{\mathrm{d} \boldsymbol{r}}{\mathrm{d} t}=v_{0} \boldsymbol{e}+\boldsymbol{F} / \gamma+\boldsymbol{\eta},
$$

where the force $\boldsymbol{F}$ between the particles is purely steric and is calculated from a Weeks-Chandler-Anderson potential $^{41}$. Here, $\boldsymbol{\eta}$ is a Gaussian white noise with zero mean and $\left\langle\boldsymbol{\eta}(t) \boldsymbol{\eta}\left(t^{\prime}\right)\right\rangle=2 D \mathbf{1} \delta\left(t-t^{\prime}\right)$, where $D$ is the translational diffusion coefficient. The motion of the orientational degrees of freedom is determined by the equation

$$
\frac{\mathrm{d} e}{\mathrm{~d} t}=\boldsymbol{\zeta} \times \boldsymbol{e},
$$

where rotational diffusion is included by the Gaussian white noise $\boldsymbol{\zeta}$ with $\left\langle\boldsymbol{\zeta}(t) \boldsymbol{\zeta}\left(t^{\prime}\right)\right\rangle=2 D_{r} \mathbf{1} \delta\left(t-t^{\prime}\right)$ and the rotational diffusion coefficient is given by $D_{r}=3 D / \sigma^{2}$. Also for the ABP model, we use a three dimensional cubic domain with periodic boundary conditions.

We note that it may be reasonable to compare our biological microswimmer model with an ABP with the same 
asymmetric shape. However, the spherical ABP model has become a canonical model used in a large fraction of the active particles literature, and a test-bed of current theories of active motion. Thus, we think that a comparison with spherical ABPs will provide more useful information.

\section{Dimensionless parameters}

For both our stroke-averaged biological microswimmer and ABP we simulate $N=300-1350$ active swimmers, resulting in filling fractions from $\phi=0.05$ to $\phi=0.25$, at a fixed volume $(100 a)^{3}$. A dimensionless quantification of the relative importance of the self-propulsion speed to diffusive processes is the Péclet number, which we define as $\mathcal{P}=v_{\text {eff }} \sigma / D$, where $v_{\text {eff }}$ and $\sigma$ are the effective speed and the effective size of the swimmer, respectively, and $D$ the diffusion constant of the swimmer (for ABP $v_{\text {eff }}=v_{0}$, whereas for the stroke-averaged biological microswimmer see $^{1}$.) We present results at $\mathcal{P} \approx 1.9 \times 10^{3}$ and $\mathcal{P} \approx 6 \times 10^{2}$. The Reynolds number of the flow around our swimmers (measuring the ratio of inertial to viscous forces) is given by $\mathcal{R}=\sigma v_{\text {eff }} \rho / \eta$, where $\rho$ and $\eta$ are the MPCD fluid's density and viscosity, respectively. The simulated Reynolds numbers are $\mathcal{R}=0.06$ for high Péclet number $\left(\mathcal{P} \approx 1.9 \times 10^{3}\right)$ and $\mathcal{R}=0.02$ at lower Péclet number $\left(\mathcal{P} \approx 6 \times 10^{2}\right)$. The corresponding force on each particle inside the regularized force region for the puller-type swimmers is $f_{0}=11.25 k_{B} T / a$ for high Péclet number $\left(\mathcal{P} \approx 1.9 \times 10^{3}\right)$ and $f_{0}=3.75 k_{B} T / a$ at lower Péclet number $\left(\mathcal{P} \approx 6 \times 10^{2}\right)$. For the pusher-type swimmers the force is $f_{0}=37.5 k_{B} T / a$ for high Péclet number $\left(\mathcal{P} \approx 1.9 \times 10^{3}\right)$ and $f_{0}=12.5 k_{B} T / a$ at lower Péclet number $\left(\mathcal{P} \approx 6 \times 10^{2}\right)$.

\section{PAIR DISTRIBUTION FUNCTION}

On very general grounds, the complete information of a liquid's structure is encapsulated in the angular pair distribution function $g\left(\boldsymbol{r}_{\mu \nu}, \boldsymbol{e}_{\mu}, \boldsymbol{e}_{\nu}\right)$ of two particles $\mu$ and $\nu$, which depends on their relative position $\boldsymbol{r}_{\mu \nu}=\boldsymbol{r}_{\mu}-\boldsymbol{r}_{\nu}$ as well as on their orientations $\boldsymbol{e}_{\mu}, \boldsymbol{e}_{\nu}{ }^{17,18}$. However, in the general case the arguments of the pair distribution function define a highly dimensional space and it has thus been proven useful to restrict oneself to a partial descriptions of the orientational ordering ${ }^{17,18}$. Since our active swimmers self-propel into the direction of their orientation $\boldsymbol{e}_{\mu}$, we expect non-trivial correlations mainly with respect to this axis. Therefore, we compute the correlation of a particle's orientation with respect to the relative position of all other particles, as illustrated in Fig. 2.

In practice, we use the following definition of our orientation-dependent pair distribution function: given two particles $\mu$ and $\nu$ with relative position $\boldsymbol{r}_{\mu \nu}=\boldsymbol{r}_{\mu}-\boldsymbol{r}_{\nu}$ and orientations $\boldsymbol{e}_{\mu}, \boldsymbol{e}_{\nu}$, we compute the distance $r=$ $\left|\boldsymbol{r}_{\mu \nu}\right|$ and the polar angle $\theta=\boldsymbol{e}_{\mu} \cdot \boldsymbol{r}_{\mu \nu} /\left|\boldsymbol{r}_{\mu \nu}\right|$ with respect to the orientation of particle $\mu$ (Fig. 2). The average over the ensemble, polar angle, and orientation of the neighboring swimmer $\boldsymbol{e}_{\nu}$ is obtained from a histogram $h\left(r_{b}, \theta_{b}\right)$ for all $N^{2}$ pairs of swimmers, where $r_{b}$ is the radial bin and $\theta_{b}$ the polar bin. The orientation-dependent pair distribution function is then given by

$$
g\left(r_{b}, \theta_{b}\right)=\frac{h\left(r_{b}, \theta_{b}\right)}{N^{2} n\left(r_{b}, \theta_{b}\right)},
$$

where $n\left(r_{b}, \theta_{b}\right)$ is the normalization

$$
\begin{aligned}
n\left(r_{b}, \theta_{b}\right)= & \frac{2}{3} \pi\left[\left(r_{b}+\delta r\right)^{3}-\left(r_{b}\right)^{3}\right] \\
& \times\left[\cos \left(\theta_{b}\right)-\cos \left(\theta_{b}+\delta \theta\right)\right],
\end{aligned}
$$

and where $\delta r$ is the bin size in the radial component and $\delta \theta$ is the bin size in the polar component. It should be noted that for a homogeneous configuration $g(r, \theta)$ is equal to one. Physically, $g(r, \theta)$ is related to the probability of finding a neighboring particle at distance $r$ and with a polar angle $\theta$ with respect to the reference particle and its orientation.

In addition to the orientation-dependent pair distribution function we calculate the radial distribution function $^{17,19}$ given by

$$
g(r)=\int_{0}^{\pi} g(r, \theta) \mathrm{d} \theta
$$

Furthermore, as an effective measure of the local structure around a microswimmer, we consider the coordination number ${ }^{19}$, which is the average number of nearest neighbors of a particle

$$
C=\int_{0}^{R_{\min }} g(r) \mathrm{d} r
$$

where $R_{\min }$ is the position of the first minimum of $g(r)$.

\section{RESULTS}

Figure 3 shows a matrix chart of our calculations of [Eq. (3)]. Different rows represent different Péclet numbers for both puller- and pusher-type swimmers, whereas the filling fraction changes with the column. First of all, we note that low filling fractions are characterized by a complex texture of peaks and minima of $g(r, \theta)$. These are stationary-state structures, and not transient, as explicitly verified in our simulations.

Pushers have increased probability to find neighbors in the frontal and rear polar regions of their bodies, while around their equator neighboring swimmers tend to be depleted. As the Péclet number increases, the asymmetry between front and back poles increases, and the rear tail region grows more populated than the front. Pullers present the most complex texture at low filling fractions. A preference for an accumulation of probability in the 


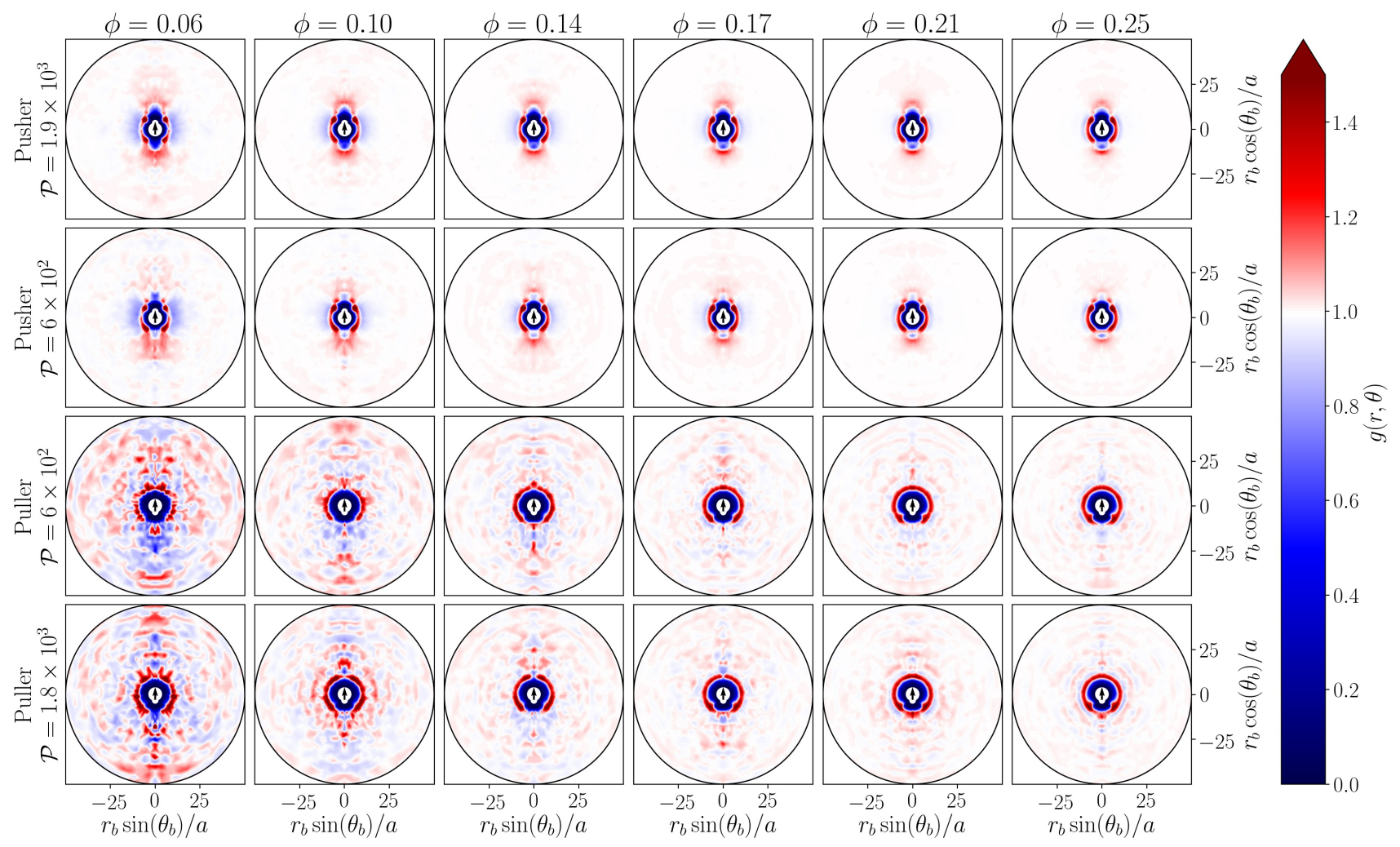

FIG. 3. Orientation-dependent pair distribution function $g(r, \theta)$ for pushers and pullers separated by a distance $r$ and with polar angle $\theta$. Each row shows a different Péclet number $\mathcal{P}$ and each column a different filling fraction $\phi$. The color encodes the value of $g(r, \theta)$, where white indicates the bulk average $g(r, \theta)=1$. The central, white dumbbells mark the position of the active swimmers and the black arrows the swimming direction.

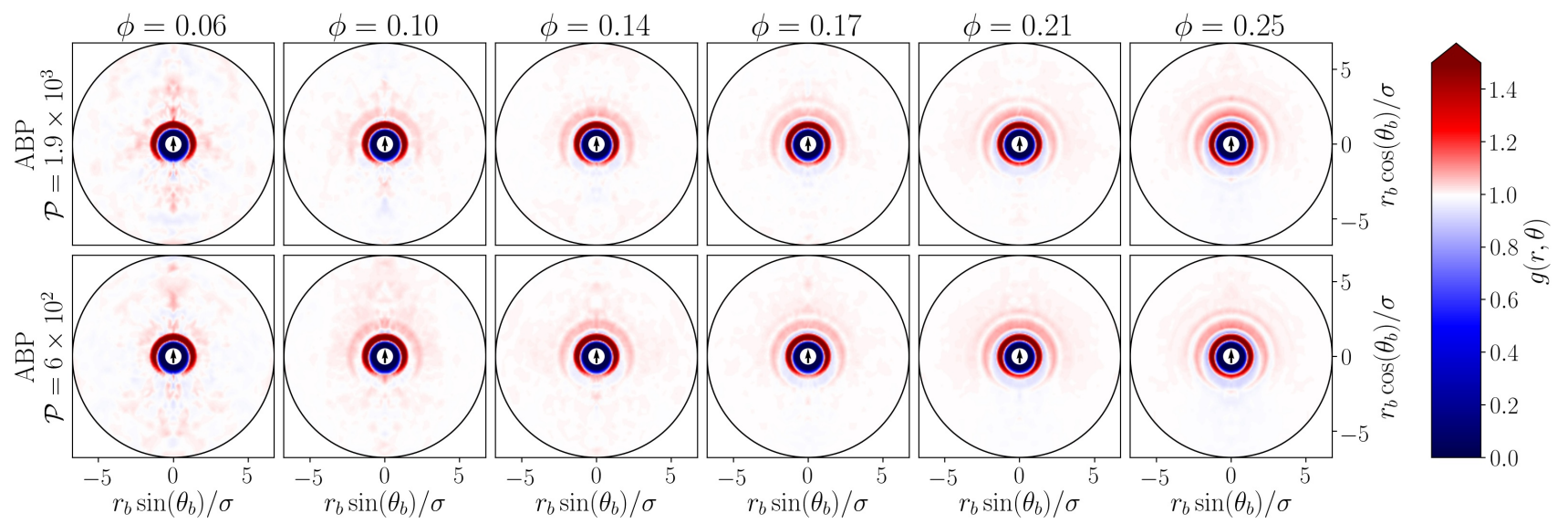

FIG. 4. Orientation-dependent pair distribution function $g(r, \theta)$ for ABP separated by a distance $r$ and with polar angle $\theta$. Each row shows a different Péclet number $\mathcal{P}$ and each column a different filling fraction $\phi$. The color encodes the value of $g(r, \theta)$, where white indicates the bulk average $g(r, \theta)=1$. The central, white circles mark the position of the ABP and back arrows the swimming direction.

front pole and in the rear region at intermediate latitudes is, however, discernible. Furthermore, at low filling fraction, $g(r, \theta)$ for both pullers and pushers shows a rather long-ranged structure, extending more than 5 times the radius of the individual swimmer.
As the filling fraction increases, $g(r, \theta)$ loses structure for both pushers and pullers at all Péclet numbers. For pushers, perpendicular to the swimming direction the pair distribution function first increases to $g(r, \theta)>1$ and then decreases to values $g(r, \theta)<1$. Furthermore, on the 
sides of the pushers one observes a band-like structure for $\phi \gtrsim 0.10$ and a quadrupolar structure for $\phi=0.06$. The increased values of $g(r, \theta)$ in front and back poles of pushers become more concentrated in smaller areas as the filling fraction is increased.

For pullers, we observe that as $\phi$ increases, $g(r, \theta)>$ 1 exhibits a ring-like structure of increased probability close to the swimmers. Furthermore, we find two distinct regions at intermediate latitudes in the rear region of the swimmer, within the ring-like structure, that show very large values of $g(r, \theta)$.

To disentangle the effects of activity, steric and hydrodynamic interactions on the pair distribution function, we performed additional simulations of $\mathrm{ABP}^{37-40}$, which only include steric interactions among swimmers. Figure 4 shows the orientation-dependent pair distribution function of ABP at different Péclet numbers and filling fractions. Even from a cursory inspection it is visible that the complex texture present at lower $\phi$ has nearly vanished. In its stead, a partial ring (interrupted in the lower pole) of pronounced peak of $g(r, \theta)$ emerges. A clear increase to $g(r, \theta)>1$ in front and a clear decrease to to $g(r, \theta)<1$ behind the ABP can be seen. This asymmetry is an effect of the self-propulsion of the ABPs and has also been studied in ${ }^{20-23}$.

The comparison of ABPs to our active swimmer model shows that the hydrodynamic interactions have a strong impact on the pair distribution function. The longranged structures that are observed in Fig. 3 at low filling fractions are not present for ABPs in Fig. 4, and thus we conclude that these are a consequence of the hydrodynamic interactions between swimmers.

At high filling fractions, however, the opposite effect is observed: ABPs show more structure than our biological swimmer model, with a second and third order of rings of $g(r, \theta)>1$ in front and extending to the sides of the ABP. Again, this a consequence of hydrodynamic interactions.

The regions of increased probability $[g(r, \theta)>1]$ around the equator of pushers, and in the rear region at intermediate latitudes for pullers strongly correlate with the structure of the respective flow fields. For pushers, the streamlines generated by a swimmer will bring a neighboring swimmer to its side [see Fig. 5(a)]. For pullers, the stagnation point in the rear region at intermediate latitudes correlates well with the region of enhanced $g(r, \theta)$ [see Fig. 5(b)]. The strong influence from the specific flow field has also been recognized in ${ }^{28}$ in the context of a minimal microswimmer model.

The asymmetric shapes of our puller- and pusher-type swimmers have minor, local effect on the structure of the pair distribution functions. For both puller- and pusher-type swimmers the shape of the depleted regions $[g(r, \theta)=0]$ around the swimmers clearly resembles the swimmers shape, as is to be expected. For puller-type swimmers the asymmetric ring-like structure region of $g(r, \theta)>1$ is induced by the asymmetric shape and increases in importance as the filling fraction increases. In comparison, the ABPs show a circular ring-like structure

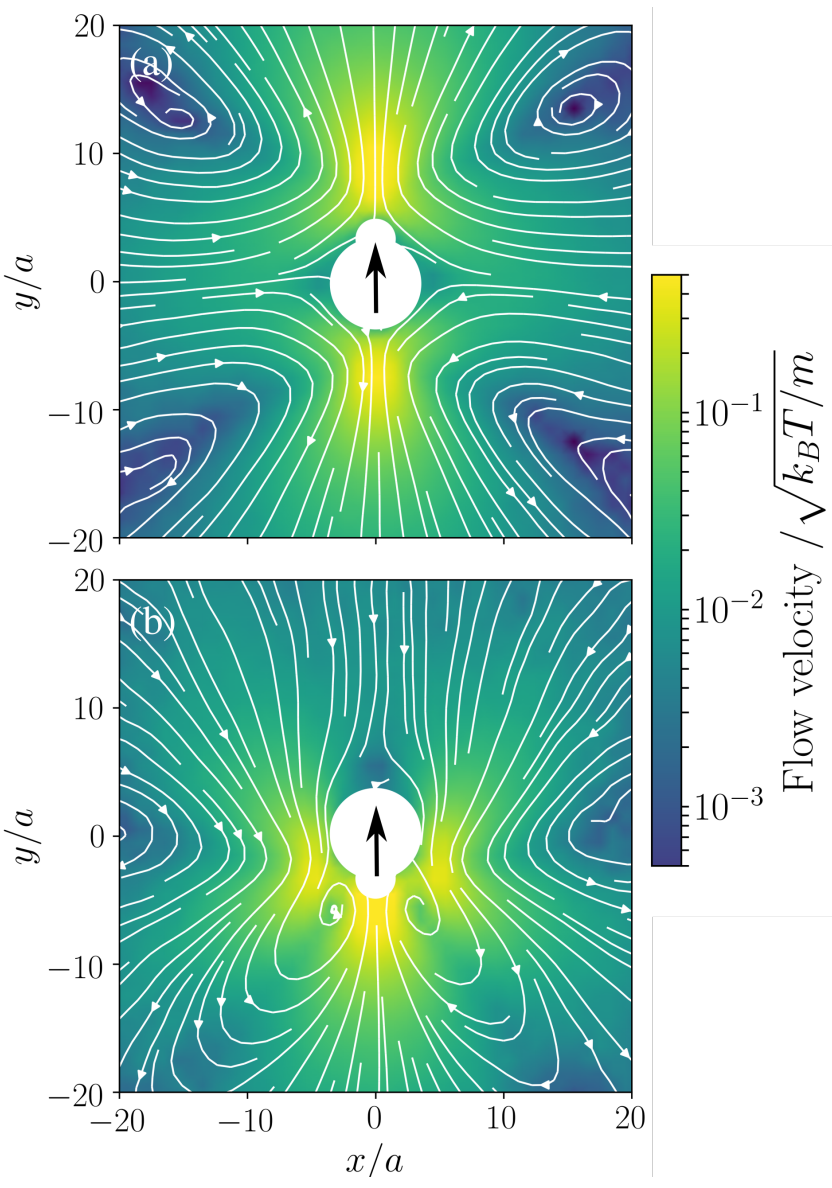

FIG. 5. Flow field of an isolated (a) pusher and (b) puller. Color code shows the flow velocity and white lines with arrows show the hydrodynamic streamlines. The white dumbbells mark the position of the swimmers and the black arrow shows the swimming direction.

that is depleted behind the swimmer, and that is induced by steric interactions and activity. For pusher-type swimmers we find that the asymmetric shape has little effect; the local structure of $g(r, \theta)$ is dominated by the hydrodynamic flow field as can be seen by the comparison of Fig. 5(a) with the distribution functions in Fig. 3.

A comparison of the results for (symmetric) active Brownian disks ${ }^{31}$ with asymmetric active self-propelled particles $^{42}$ reveals only quantitative changes in the structure factor, but no qualitative difference. This shows that an asymmetric shape has only minor effects on the structural properties, and thus on the pair distribution function, at least at the filling fractions explored here. Again, a comparison to our results highlights the importance of the hydrodynamic interactions between microswimmers for the radial distribution function.

Although the orientation-dependent pair distribution function already contains a coarse-grained level of description, complex structures are visible. It proves useful to observe an even more coarse-grained quantity $g(r)$, obtained from averaging over the polar angle $\theta$ and that 


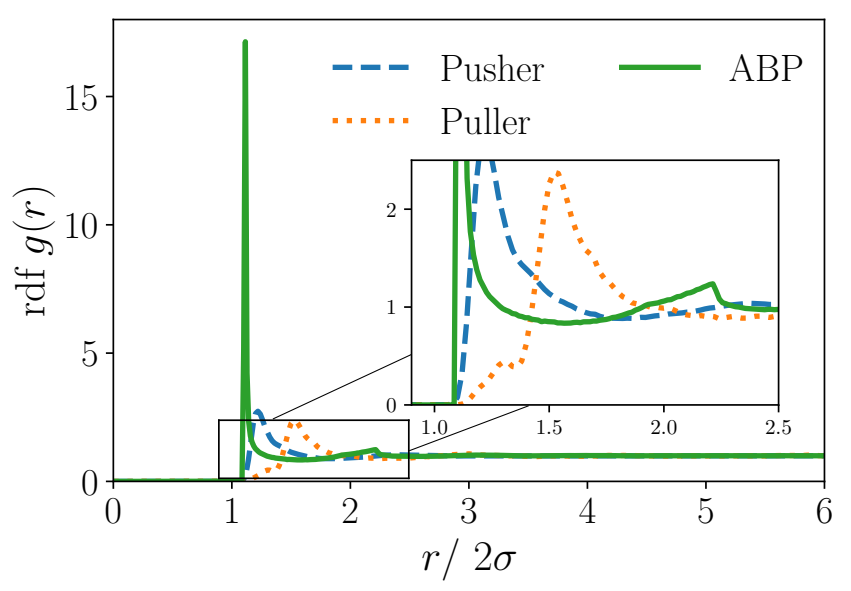

FIG. 6. Radial distribution function for ABP, pushers, and pullers at filling fraction $\phi=0.25$ and Péclet number $\mathcal{P}=$ $6 \times 10^{2}$. The inset shows a zoom in of the radial distribution function in the first peak region. The $r$-axis is scales by the typical particle diameter $2 \sigma$ (for pullers and pushers we use $\sigma=5 a)$

will capture the average environment experienced by a swimmer over a long period of time. Figure 6 shows the radial distribution function $g(r)$ for pushers, pullers, and ABPs. ABPs exhibit a remarkably pronounced first peak and a much weaker second peak; a similar structure for $\mathrm{ABP}$ was also found by ${ }^{31,43}$.

The first shell of neighbors for both pushers and pullers is considerably weaker than the ABP's. This is a combination of the hydrodynamic interactions that quickly reorient particles, and the typically long reorientation times of ABP combined with steric effects ${ }^{44}$. Even at this considerable level of coarse-graining, it is visible how the different flow fields of pushers and pullers generate different structures in $g(r)$. Pushers show only a single peak, whereas pullers have a weak first and a stronger second peak. Comparing pushers and pullers with ABPs reveals that hydrodynamic interactions drastically reduce the height of the first peak but enhance its width.

To study the nearest-neighbor environment in more detail, we report the dependence of the coordination number $C$ on the filling fraction $\phi$ in Fig. 7. As $\phi$ increases, $C$ increases monotonically for pushers, pullers, and ABP at both Péclet numbers investigated. However, for ABPs the coordination number is always larger than for pushers and pullers, confirming the result that the hydrodynamic interactions decrease the number of nearest neighbors.

\section{CONCLUSIONS}

We have presented the orientation-dependent pair distribution function $g(r, \theta)$ for hydrodynamically interacting active swimmers at different Péclet numbers and a range of filling fractions. To disentangle the effects for

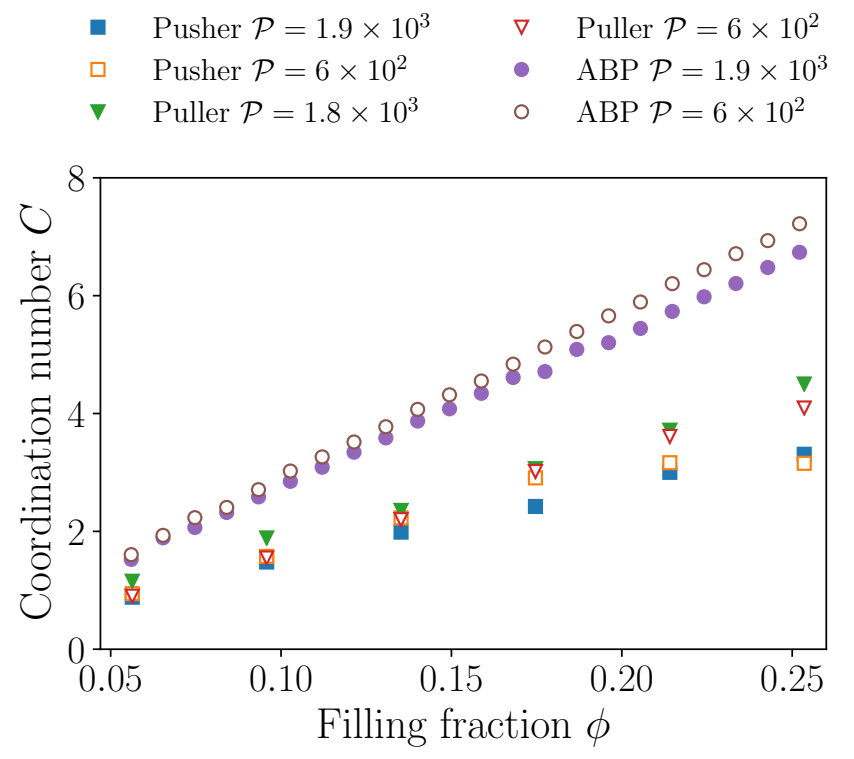

FIG. 7. Dependence of the coordination number $C$ on the filling fraction $\phi$. Pushers (squares), pullers (triangles) and ABP (circles) are shown for two different Péclet numbers.

activity, steric and hydrodynamic interactions we compared our results to simulations of active Brownian particles. We found that the hydrodynamic interactions introduce long-ranged structures into the orientationdependent pair correlation function at low filling fractions. As the filling fraction increases, hydrodynamic interactions suppress the complex texture of $g(r, \theta)$. In fact, the influence of hydrodynamic interactions on the orientation-dependent pair correlation function is strong and the specific flow field introduces a non-trivial asymmetry in the $g(r, \theta)$ for both pushers and pullers.

Investigation of the radial distribution function for pushers, pullers, and active Brownian particles shows that hydrodynamic interactions strongly suppress the height of the first peak and also broaden it. As an effective measure of the average immediate neighborhood of a given microswimmer, the coordination number reveals that hydrodynamic interactions among microswimmers suppress the strong first shell of neighbors.

Our present work lays the foundations for a theoretical investigation of the complex interplay of hydrodynamics, steric forces, and active motion. An open - and ambitious - question is whether knowledge of the active liquid's structure is sufficient for the development of a nonequilibrium statistico-mechanical theory.

\section{CONFLICTS OF INTEREST}

There are no conflicts of interest to declare. 


\section{ACKNOWLEDGEMENTS}

We gratefully acknowledge support from the Deutsche Forschungsgemeinschaft (SFB 937, project A20).

${ }^{1}$ F. J. Schwarzendahl and M. G. Mazza, Soft Matter 14, 4666 (2018).

${ }^{2}$ C. Dombrowski, L. Cisneros, S. Chatkaew, R. E. Goldstein, and J. O. Kessler, Phys. Rev. Lett. 93, 098103 (2004).

${ }^{3}$ T. Ostapenko, F. J. Schwarzendahl, T. J. Böddeker, C. T. Kreis, J. Cammann, M. G. Mazza, and O. Bäumchen, Phys. Rev. Lett. 120, 068002 (2018).

${ }^{4}$ E. Lauga, W. R. DiLuzio, G. M. Whitesides, and H. A. Stone, Biophys. J. 90, 400 (2006).

${ }^{5}$ A. P. Berke, L. Turner, H. C. Berg, and E. Lauga, Phys. Rev. Lett. 101, 038102 (2008).

${ }^{6}$ H. H. Wensink, J. Dunkel, S. Heidenreich, K. Drescher, R. E. Goldstein, H. Löwen, and J. M. Yeomans, Proc. Natl. Acad. Sci. USA 109, 14308 (2012).

${ }^{7}$ M. F. Copeland and D. B. Weibel, Soft Matter 5, 1174 (2009).

${ }^{8}$ H. Wioland, F. G. Woodhouse, J. Dunkel, J. O. Kessler, and R. E. Goldstein, Phys. Rev. Lett. 110, 268102 (2013).

${ }^{9}$ J. J. Molina, Y. Nakayama, and R. Yamamoto, Soft Matter 9, 4923 (2013).

${ }^{10}$ A. Zöttl and H. Stark, Phys. Rev. Lett. 112, 118101 (2014).

${ }^{11}$ A. A. Evans, T. Ishikawa, T. Yamaguchi, and E. Lauga, Phys. Fluids 23, 111702 (2011).

${ }^{12}$ H. Wioland, E. Lushi, and R. E. Goldstein, New J. Phys. 18, 075002 (2016).

${ }^{13}$ D. L. Koch and G. Subramanian, Annual Review of Fluid Mechanics 43, 637 (2011).

${ }^{14}$ M. C. Marchetti, J.-F. Joanny, S. Ramaswamy, T. B. Liverpool, J. Prost, M. Rao, and R. A. Simha, Reviews of Modern Physics 85, 1143 (2013).

${ }^{15}$ M. Enculescu and H. Stark, Phys. Rev. Lett. 107, 058301 (2011).

${ }^{16} \mathrm{~J}$. Vachier and M. G. Mazza, The European Physical Journal E 42, 11 (2019).

${ }^{17}$ M. P. Allen and D. J. Tildesley, Computer Simulation of Liquids (Oxford University Press, 1989).

${ }^{18}$ C. Gray and K. Gubbins, Clarendon, Oxford (1984).

${ }^{19}$ J.-P. Hansen and I. R. McDonald, Theory of simple liquids (Elsevier, 1990).

${ }^{20}$ J. Bialké, H. Löwen, and T. Speck, EPL (Europhysics Letters) 103, 30008 (2013).

${ }^{21}$ A. Härtel, D. Richard, and T. Speck, Physical Review E 97, 012606 (2018).

${ }^{22}$ C. Hoell, H. Löwen, and A. M. Menzel, The Journal of chemical physics 149, 144902 (2018).
${ }^{23}$ R. Wittkowski, J. Stenhammar, and M. E. Cates, New Journal of Physics 19, 105003 (2017).

${ }^{24}$ I. H. Riedel, K. Kruse, and J. Howard, Science 309, 300 (2005).

${ }^{25}$ F. Alarcón and I. Pagonabarraga, J. Mol. Liq. 185, 56 (2013).

${ }^{26}$ F. Kogler and S. H. Klapp, EPL (Europhysics Letters) 110, 10004 (2015).

${ }^{27}$ W. Yang, V. R. Misko, J. Tempere, M. Kong, and F. M. Peeters, Physical Review E 95, 062602 (2017).

${ }^{28}$ G. Pessot, H. Löwen, and A. M. Menzel, Molecular Physics 116, 3401 (2018).

${ }^{29}$ E. Locatelli, F. Baldovin, E. Orlandini, and M. Pierno, Physical Review E 91, 022109 (2015).

${ }^{30}$ M. Theers, E. Westphal, K. Qi, R. G. Winkler, and G. Gompper, Soft matter (2018).

${ }^{31}$ N. de Macedo Biniossek, H. Löwen, T. Voigtmann, and F. Smallenburg, Journal of Physics: Condensed Matter 30, 074001 (2018).

${ }^{32}$ G. Gompper, T. Ihle, D. M. Kroll, and R. G. Winkler, Multiparticle collision dynamics: a particle-based mesoscale simulation approach to the hydrodynamics of complex fluids, Vol. Advanced computer simulation approaches for soft matter sciences III (Springer, 2009) pp. 1-87.

${ }^{33}$ K. Drescher, J. Dunkel, L. H. Cisneros, S. Ganguly, and R. E. Goldstein, Proc. Natl. Acad. Sci. USA 108, 10940 (2011), http://www.pnas.org/content/108/27/10940.full.pdf.

${ }^{34}$ K. Drescher, R. E. Goldstein, N. Michel, M. Polin, and I. Tuval, Phys. Rev. Lett. 105, 168101 (2010).

${ }^{35}$ H. Noguchi, N. Kikuchi, and G. Gompper, Europhys. Lett. 78, 10005 (2007).

${ }^{36}$ I. O. Götze, H. Noguchi, and G. Gompper, Phys. Rev. E 76, 046705 (2007).

${ }^{37}$ A. Wysocki, R. G. Winkler, and G. Gompper, EPL (Europhysics Letters) 105, 48004 (2014).

${ }^{38}$ Y. Fily and M. C. Marchetti, Phys. Rev. Lett. 108, 235702 (2012).

${ }^{39}$ G. S. Redner, M. F. Hagan, and A. Baskaran, Phys. Rev. Lett. 110, 055701 (2013).

${ }^{40}$ J. Bialké, T. Speck, and H. Löwen, Phys. Rev. Lett. 108, 168301 (2012).

${ }^{41}$ J. D. Weeks, D. Chandler, and H. C. Andersen, J. Chem. Phys. 54, 5237 (1971).

${ }^{42}$ H. Wensink, V. Kantsler, R. Goldstein, and J. Dunkel, Physical Review E 89, 010302 (2014).

${ }^{43}$ T. F. Farage, P. Krinninger, and J. M. Brader, Physical Review E 91, 042310 (2015).

${ }^{44}$ R. Matas-Navarro, R. Golestanian, T. B. Liverpool, and S. M. Fielding, Phys. Rev. E 90, 032304 (2014). 